\title{
Genetically encoded fluorescent sensors of membrane potential
}

\author{
B. J. Baker ${ }^{1}$, H. Mutoh ${ }^{2}$, D. Dimitrov ${ }^{2}$, W. Akemann ${ }^{2}$, A. Perron ${ }^{2}$, Y. Iwamoto ${ }^{2}$, L. Jin ${ }^{1}$, L. B. Cohen ${ }^{1}$, \\ E. Y. Isacoff ${ }^{3}$, V. A. Pieribone ${ }^{1,4}$, T. Hughes ${ }^{5}$ and T. Knöpfel ${ }^{2, *}$ \\ ${ }^{1}$ Department of Cellular and Molecular Physiology, Yale University School of Medicine, \\ New Haven, CT 06520, USA \\ ${ }^{2}$ Laboratory for Neuronal Circuit Dynamics, Brain Science Institute, RIKEN, 2-1 Hirosawa, \\ Wako-Shi, Saitama 351-0198, Japan (*author for correspondence; e-mail: knopfel@brain.riken.go.jp) \\ ${ }^{3}$ Department of Molecular and Cell Biology, University of California Berkeley, Berkeley, CA 94720, USA \\ ${ }^{4}$ John B. Pierce Laboratory, New Haven, CT 06520, USA \\ ${ }^{5}$ Department of Cell Biology and Neuroscience, Montana State University, Bozeman, MT 59717, USA
}

Received 1 April 2008; Revised 26 May 2008; Accepted 6 June 2008

Published online 5 August 2008

(c) The Author(s) 2008. This article is published with open access at Springerlink.com

Imaging activity of neurons in intact brain tissue was conceived several decades ago and, after many years of development, voltage-sensitive dyes now offer the highest spatial and temporal resolution for imaging neuronal functions in the living brain. Further progress in this field is expected from the emergent development of genetically encoded fluorescent sensors of membrane potential. These fluorescent protein (FP) voltage sensors overcome the drawbacks of organic voltage sensitive dyes such as non-specificity of cell staining and the low accessibility of the dye to some cell types. In a transgenic animal, a genetically encoded sensor could in principle be expressed specifically in any cell type and would have the advantage of staining only the cell population determined by the specificity of the promoter used to drive expression. Here we critically review the current status of these developments.

\section{Introduction}

Optical imaging is a remarkably flexible method for studying various cellular activities. Organic dyes have been developed that can faithfully report many biological variables including calcium concentration, $\mathrm{pH}$, or membrane potential (Davila et al., 1973; Brown et al., 1975; MacDonald and Jobsis, 1976). These optical probes enable simultaneous measurements from many locations and have been used to study the physiology of single

B. J. Baker, H. Mutoh, D. Dimitrov, and W. Akemann contributed equally to this article. neurons as well as large populations of cells (selected work reviewed in Grinvald and Hildesheim, 2004; Baker et al., 2005; and Knöpfel et al., 2006).

Two major drawbacks of these organic dyes are the non-specificity of cell staining and the low accessibility of the dye to some cell types. They either stain all cells in a tissue to which they are exposed, or if delivered in a patch pipette they can only label one or a small number of cells. In the absence of targeting of the organic dyes to specific cell types, optical signals of interest are often drowned out either by background fluorescence from inactive cells or by signals in cells that are not the focus of interest. In addition, with the exception of preparations with sparse cell staining, diffuse labeling limits the spatial resolution so that single 
cell responses cannot be readily resolved. One general solution to this problem is to make optical reporters from proteins. Since protein-based reporters are encoded in DNA, they can be placed under the control of cell-specific promoters and introduced in vivo using gene transfer techniques. Moreover, protein-based reporters can, in principle, be rationally "tuned" by modification of their functional domains with mutations that adjust their dynamic range of operation. In a transgenic animal, a genetically encoded sensor could in principle be expressed in any cell type and would have the advantage of staining only the cell population determined by the specificity of the promoter used to drive expression.

Protein-based reporters are generally constructed from two parts: a "sensor" protein that undergoes a conformational rearrangement that depends on the parameter measured (e.g., a voltage-gated ion channel that senses membrane potential), and a fluorescent protein (FP) reporter fluorophore whose optical output is modulated by the sensor protein (for a recent review, see Knöpfel et al., 2006). The first FP voltage sensor, denoted FlaSh, was obtained by inserting GFP downstream of the pore region in the Drosophila voltage-gated potassium channel, Shaker (Siegel and Isacoff, 1997). When FlaSh was expressed in Xenopus oocytes, changes in membrane potential were reported by changes of the fluorescence. However, this initial success was not followed by published reports of signals from mammalian brains using FlaSh or the two subsequent first generation voltage sensors, VFSP-1 (Sakai et al., 2001; Knöpfel et al., 2003) and SPARC (Ataka and Pieribone, 2002).

A group of laboratories (Yale, PI Cohen; Berkeley, PI Isacoff; Yale, PI Pieribone; Montana, PI Hughes; and RIKEN BSI, PI Knöpfel) has begun an effort to improve genetically encoded FP voltage sensors for use in mammalian preparations. In order to realize a new, improved generation of sensors, inefficient targeting to mammalian plasma membrane was identified as a major limitation of the first generation FP voltage sensors. Therefore, the group started a concerted effort to find a more suitable signaling protein and FP reporter-combination, whilst also fine-tuning emerging sensor proteins.

Here we review the historical development of the first generation FP voltage sensors, describe the state-of-the-art second generation FP voltage sensors, and outline efforts for the next generation
FP voltage sensors. We then compare these traditional fully genetically encodable sensors with new partially genetically encoded probes. Finally, we critically discuss the practical and theoretical limitations of current FP voltage sensor-based imaging techniques.

\section{First generation FP voltage sensors}

The first generation FP voltage sensors were developed by molecular fusion of a GFP-based fluorescent reporter to voltage-gated ion channels or components thereof. There are three prototypes obtained from three different laboratories, each of which constitutes a proof of principle and provided valuable insights (Fig. 1).

The very first prototype, FlaSh, was generated in the Isacoff laboratory and obtained by fusing wtGFP to the C-terminus of the Drosophila Shaker potassium channel (Siegel and Isacoff, 1997). When expressed in oocytes, an 80-mV depolarization of the plasma membrane resulted in a $5 \%$ decrease in fluorescence (Fig. 1 left panel), this was designated FlaSh, for "fluorescent Shaker".

To reduce unwanted effects on the cell's physiology, FlaSh was rendered non-conducting by introducing a W434F mutation, preventing ions from moving through the pore while maintaining voltage-dependent rearrangements (Perozo et al., 1993). In order to resolve action potentials, an ideal sensor would generate a robust signal on a millisecond timescale. The signal strength from FlaSh is comparable to that of the voltage-sensitive organic dye, di4-ANEPPS; however, the on and off rates are rather slow ( $\tau$-on $\sim 100 \mathrm{~ms} ; \tau$-off $\sim 60 \mathrm{~ms}$ ). In an effort to improve the kinetics of FlaSh, Guerrero et al. (2002) replaced the wtGFP with several different FPs. The change in optical characteristics mediated by the different FPs was substantial. The signal intensity, the direction of the fluorescent change, and the speed of FlaSh were all altered by the chromophore in an unsystematic way. Variants involving wtGFP and uvGFP both have reduced fluorescence in response to depolarization steps, while Ecliptic variants of GFP, YFP, and CFP exhibit increased fluorescence in response to depolarization. Remarkably, upon depolarization, an increase in fluorescence is seen when eGFP is excited at $450 \mathrm{~nm}$, but there is a 


\section{FlaSh}
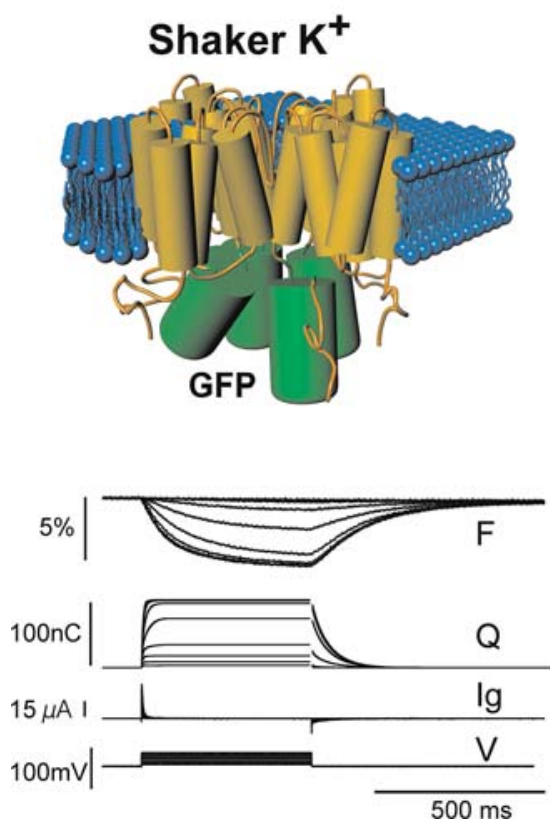

VSFP1
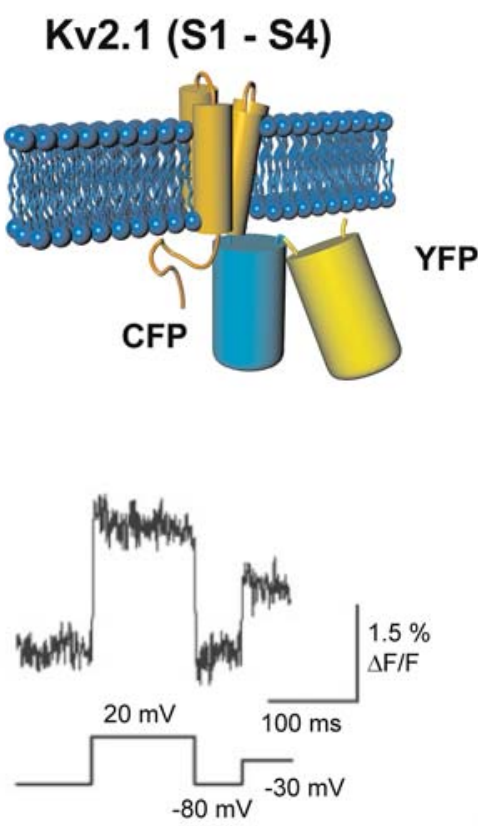

SPARC rSkM1
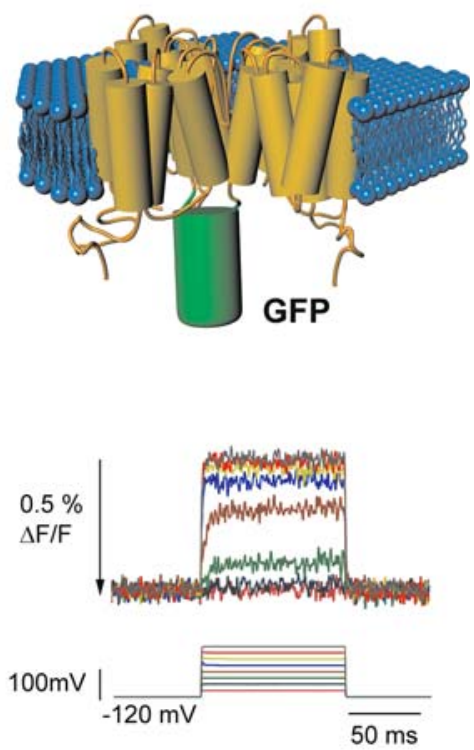

Fig. 1. Fist generation FP voltage sensors. FlaSh (left panel) was generated by fusing wtGFP to the C-terminus of Drosophila Shaker potassium channel. Simultaneous two-electrode voltage-clamp recording and photometry in Xenopus oocytes show current and fluorescence changes in response to voltage steps $(\mathrm{V})$ between -60 and $10 \mathrm{mV}$, in $10 \mathrm{mV}$ increments. Holding potential was $-80 \mathrm{mV}$. FlaSh exhibits on and off gating currents (Ig) but no ionic current. Integrating the gating current gives the total gating charge (Q) moved during the pulse. FlaSh fluorescence $(F)$ decreases reversibly in response to membrane depolarizations. Traces are the average of 20 sweeps. Modified from Siegel and Isacoff (1997). VSFP1 (middle panel) consist of an isolated voltage sensor domain coupled to a pair of cyan and yellow fluorescent proteins. Recordings were done in HEK 293 cells. Modified from Sakai et al. (2001). SPARC (right panel) was generated by inserting an FP between domains I and II of the rat skeletal muscle $\mathrm{Na}^{+}$channel. Recordings were done in Xenopus oocytes. Modified from Ataka and Pieribone (2002).

decrease in fluorescence when excited at $480 \mathrm{~nm}$. The speed of the response is also governed by the chromophore, with the Ecliptic variant of GFP generating the fastest response ( $\tau$-on $\sim 5 \mathrm{~ms})$. While the mechanism underlying the fluorescence change that results from an alteration in membrane potential remains poorly understood, it is clear that the fluorescent reporter contributes significantly to the kinetics of the optical response and is an important parameter to vary in an attempt to improve genetically encoded voltage sensors.

The second prototypic design realized in the Knöpfel laboratory and termed VSFP1 exploits the voltage-dependent conformational changes around the fourth transmembrane segment (S4) of the voltage-gated potassium channel Kv2.1 and uses either fluorescence resonance energy transfer (FRET) (Fig. 1, middle panel; Sakai et al., 2001) or a permuted FP (Knöpfel et al., 2003). The third prototype, SPARC (Pieribone laboratory; Fig. 1, right panel), was generated by inserting a FP between domains I and II of the rat skeletal muscle $\mathrm{Na}^{+}$channel (Ataka and Pieribone, 2002).

These first generation voltage sensors are capable of optically reporting changes in membrane potential, but their use in mammalian systems is significantly hindered by their poor plasma membrane expression. When expressed in HEK 293 cells, the expression of these constructs is primarily intracellular and little if any of these first generation FP voltage sensors are co-localized at the cell surface with di8-ANEPPS (Fig. 2). Figure 2B shows the profiles of the FP in green and that of di8-ANEPPS in red along the red lines in the right column of Fig. 2A. Much better co-localization occurs with Kv1.4 with GFP at the $\mathrm{N}$-terminus and with the cation/chloride cotransporter, NKCC1, with YFP fused near the carboxyl 


\section{CONFOCAL IMAGES OF HEK 293 CELLS}

\section{A Images}

\section{SPARC}

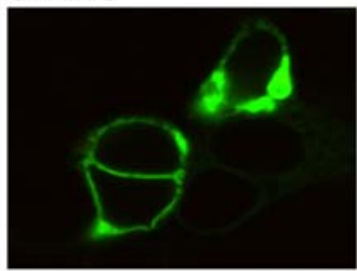

VSFP-1

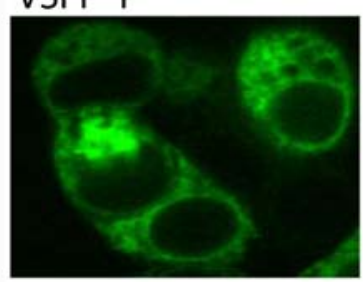

Flare

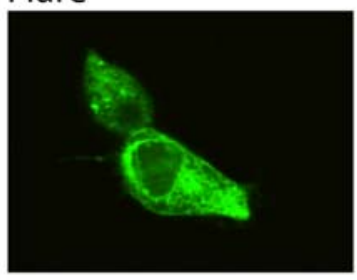

\section{Kv1.4-N-GFP}

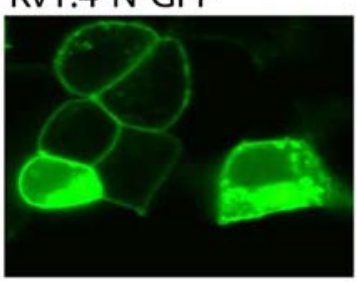

NKCC1

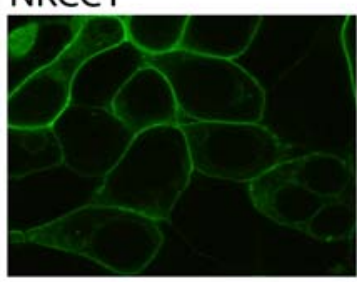

\section{B Profiles}
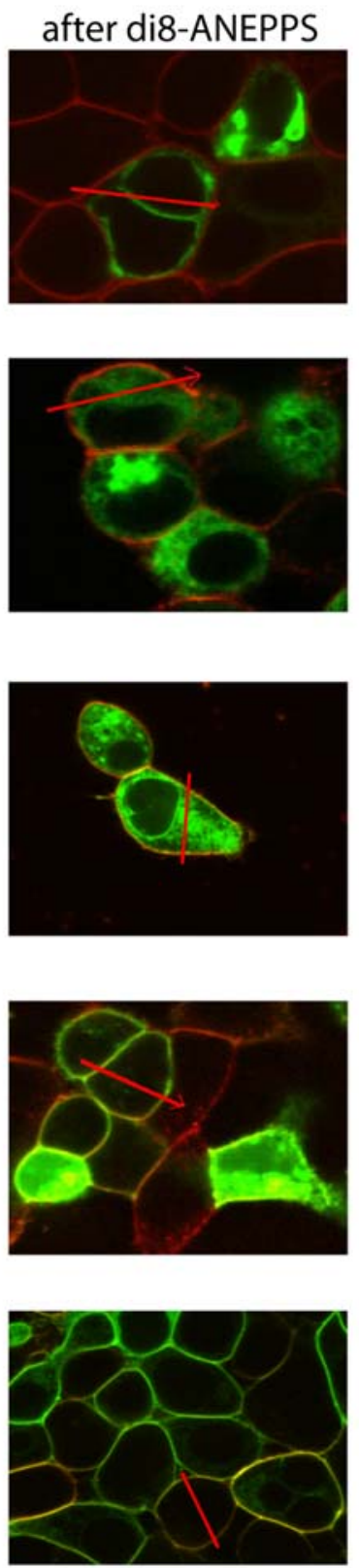
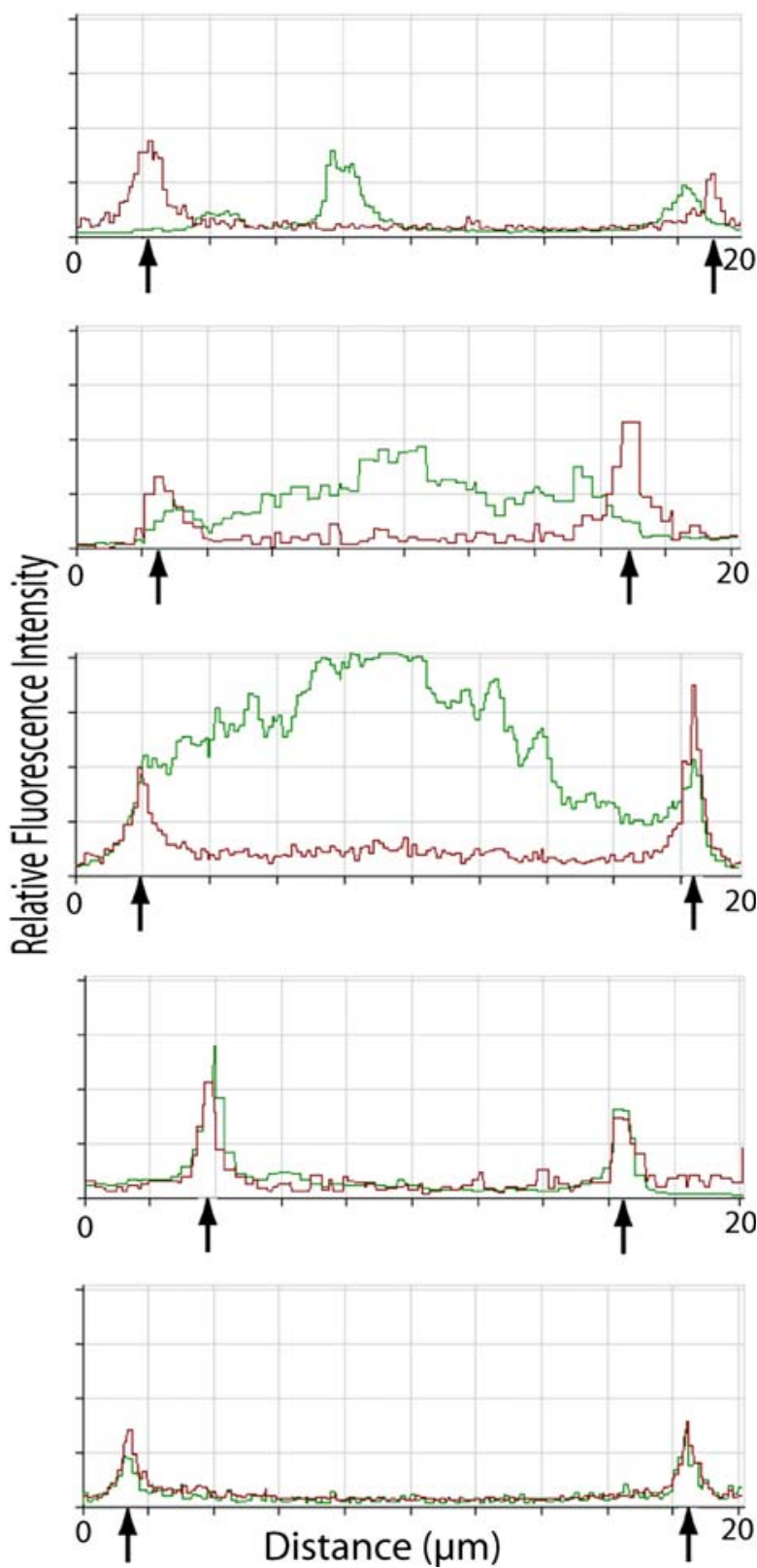

Fig. 2. Confocal images of HEK 293 cells. (A) SPARC, VSFP-1, Flare, Kv1.4-N-GFP, or NKCC1-YFP were expressed in HEK 293 cells and imaged via confocal microscopy. The images on the left show HEK 293 cells expressing the fluorescent construct. The images on the right are the same cells after the addition of di8-ANEPPS to the bathing medium. Di8-ANEPPS functions as a fluorescent plasma membrane marker. (B) The profiles show the green (FP voltage sensor) and red (di8-ANEPPS) fluorescence along the arrow in the images. The arrows indicated the location of the external membrane. Taken from Baker et al. (2007). 
terminus. There does seem to be some plasma membrane expression for Flare (a Kv1.4 variant of FlaSh), but the best-case scenario still exhibits fluorescence of a predominantly intracellular origin. More importantly, no functional optical signals could be detected using Flare, VSFP1, or SPARC (Baker et al., 2007)

The absence of a signal from the first generation probes is in part due to their low membrane expression and in part due to a large, nonresponsive background fluorescence that would mask any voltage-dependent signal. Because Kv1.4 with an N-terminal GFP exhibits excellent membrane expression, several strategies to release Flare or its Kv2.1-based homologues from the ER have been tried. Unfortunately, mutagenesis of potential ER retention signals, addition of ER release motifs, and expression in hippocampal neurons that endogenously express trafficking partners all failed to significantly improve the plasma membrane expression (Ray et al., unpublished observations; Baker and Cohen, unpublished observations).

For the second generation of FP voltage sensors, we hoped to overcome this issue of poor targeting to neuronal membranes.

\section{Second generation fP voltage sensors}

Recently, several lines of evidence supported the concept of self-contained voltage sensor domains in naturally occurring proteins such as Kv channels. Thus, a voltage sensor domain may function in the absence of the structures seen in the complete $\mathrm{Kv}$ channel complex. The first body of evidence pointing toward this possibility came from crystal structures and structure-function modeling of KvAP and $\mathrm{Kv} 1.2$ that indicated that the secondary structure of the S1-S4 portion of $\mathrm{Kv}$ channels is relatively independent from neighboring parts of these channels (Jiang et al., 2003a, b; Long et al., $2005 a, b)$. The second line of evidence was based on the identification of voltage sensor domains in non-ion channel proteins. Ci-VSP (Ciona intestinalis Voltage-Sensor-containing Phospatase), for example, is a voltage-controlled enzyme consisting of a transmembrane voltage sensor domain and a cytosolic phosphoinositide phosphatase domain (Murata et al., 2005). The voltage sensor domain from this protein was shown to be functional (i.e., able to generate gating current in response to voltage steps) when the enzyme domain was removed (Murata et al., 2005). More recently, it was also shown by the Isacoff laboratory that the Ci-VSP can exist as a monomer in the plasma membrane (Kohout et al., 2007). A third line of evidence for voltage sensor domains that can function in isolation emerged with the discovery of a new protein family termed Hv1 or voltage sensor domain only proteins (VSOPs). These membrane proteins mediate voltage-dependent proton transport and have a domain homologous to the S1-S4 portion of $\mathrm{Kv}$ channels but lack the putative pore forming S5-S6 domain (Ramsey et al., 2006; Sasaki et al., 2006). Hv1 is a dimer, with each of the voltage sensor domains containing its own gated pore; moreover, the pore can function when mutations are made that turn the channel into a monomer consisting of only a single voltage sensor domain (Tombola et al., 2008).

A self-contained voltage sensor domain that functions without additional protein components or without the need for subunit multimerization is a reasonable candidate module for an FP voltage sensor, since it avoids potential interference with assembly that may be caused by the FP. Therefore the discovery of Ci-VSP was of immediate interest. In addition, since Ci-VSP is not a channel, a sensor domain based on it would not need to be rendered non-conducting while still maintaining high expression, a challenge for channel-based sensors. Moreover, the Ci-VSP being of heterologous origin might make it less susceptible to post-translational modifications and mistargeting in mammalian expression systems. With the improvement of plasma membrane targeting in mind, the use of Ci-VSP as the basis of a sensor was explored by the Knöpfel laboratory (Dimitrov et al., 2007). Because the voltage sensor domain of Ci-VSP is homologous to that of canonical Kv channel subunits, the first step was to generate constructs similar to the first generation VSFPs (VSFP1s), but with the voltage sensor domain of Kv2.1 replaced by that of Ci-VSP. Since the exact role of the linkers coupling the Ci-VSP voltage sensor domain to the enzyme domain that it controls is unclear, the CFP and YFP FRET pair was fused to the $\mathrm{C}$-terminus of the voltage sensor domain conserving four different lengths of the intrinsic Ci-VSP S4-segment-downstream sequence. With this approach the cytosolic phosphatase domain was 

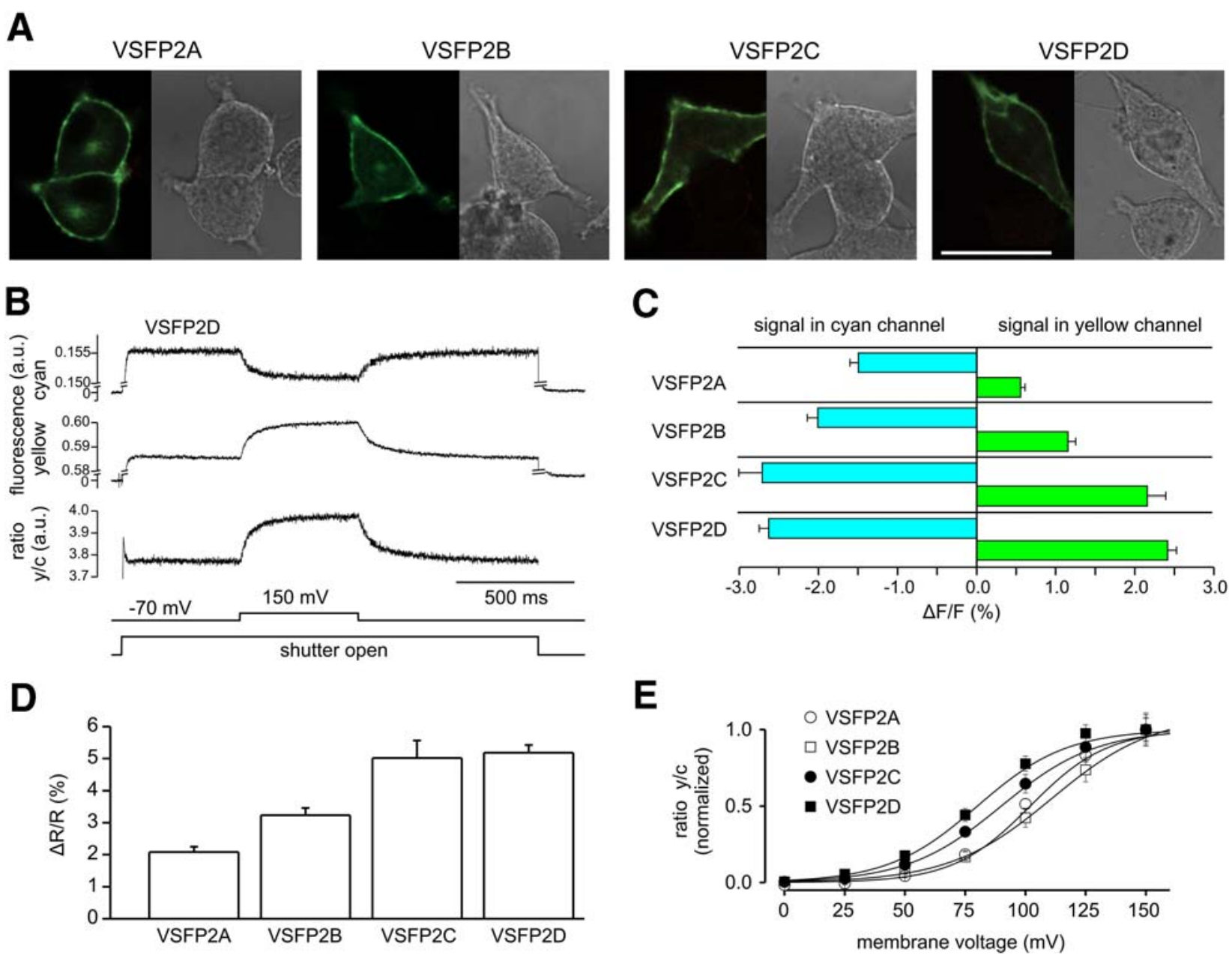

Fig. 3. Efficient plasma membrane targeting and voltage report of VSFP2s. (A) Confocal fluorescence and transmission images of PC12 cells transfected with VSFP2A, VSFP2B, VSFP2C, and VSFP2D. Scale bar is $30 \mu \mathrm{M}$. (B) Sample traces of cyan fluorescence, yellow fluorescence, and the ratio of yellow/cyan fluorescence (average of 27 traces). Lower traces indicate times of shutter opening and membrane depolarization from -70 to $150 \mathrm{mV}$. (C) Average changes in cyan fluorescence and yellow fluorescence induced by depolarization to $150 \mathrm{mV}$ in VSFP2A (11 cells), VSFP2B ( 7 cells), VSFP2C (7 cells), and VSFP2D (7 cells). (D) Average changes in the ratio of yellow and cyan fluorescence induced by depolarization to $150 \mathrm{mV}$. (E) Ratio of yellow/cyan fluorescence versus test membrane voltage. Lines are Boltzmann fits. Modified from Dimitrov et al. (2007).

essentially replaced with the FRET pair. When expressed in PC12 cells or hippocampal neurons, all of the initial series of second-generation VSFP (VSFP2) variants displayed bright fluorescence and clear targeting to the plasma membrane (Dimitrov et al., 2007). More importantly, all of these first four constructs showed voltage-dependent modulation of cyan and yellow fluorescent output (Fig. 3). The FRET fluorescence change could result from a change in the distance separating the two chromophores or from a change in the angle between them or both. Furthermore, the
Cyan signal is substantially larger than the Yellow signal for VSFP2A (Fig. 3), suggesting that nonFRET mechanisms are also occurring. At present we have no understanding of the mechanism coupling the voltage-dependent structural changes in the voltage-sensitive domain to the changes in fluorescence of the chromophores.

Consistent with "gating" currents measured in Ci-VSP, the fluorescence voltage curves for VSFP2A-D (Fig. 3, upper traces) exhibited half maximal activation $\left(V_{1 / 2}\right)$ at potentials far above the physiological range of mammalian neurons. To 
A1

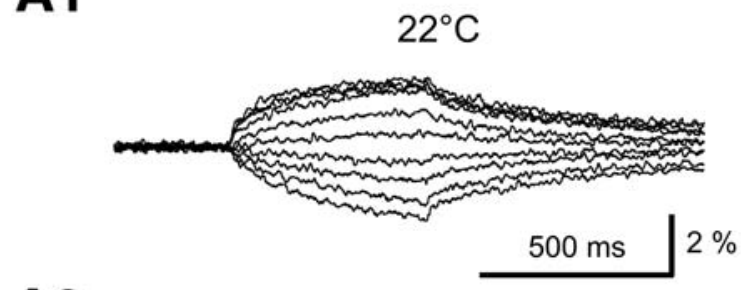

A2
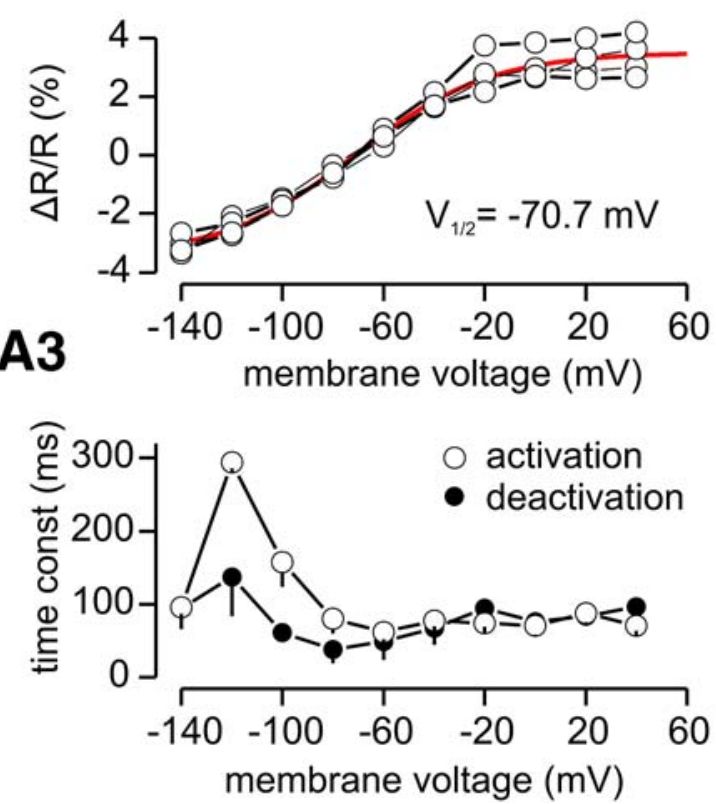

B1

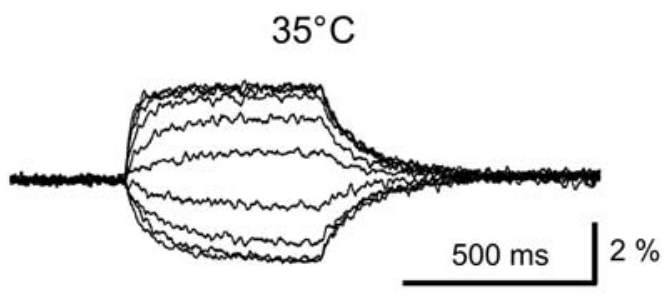

B2
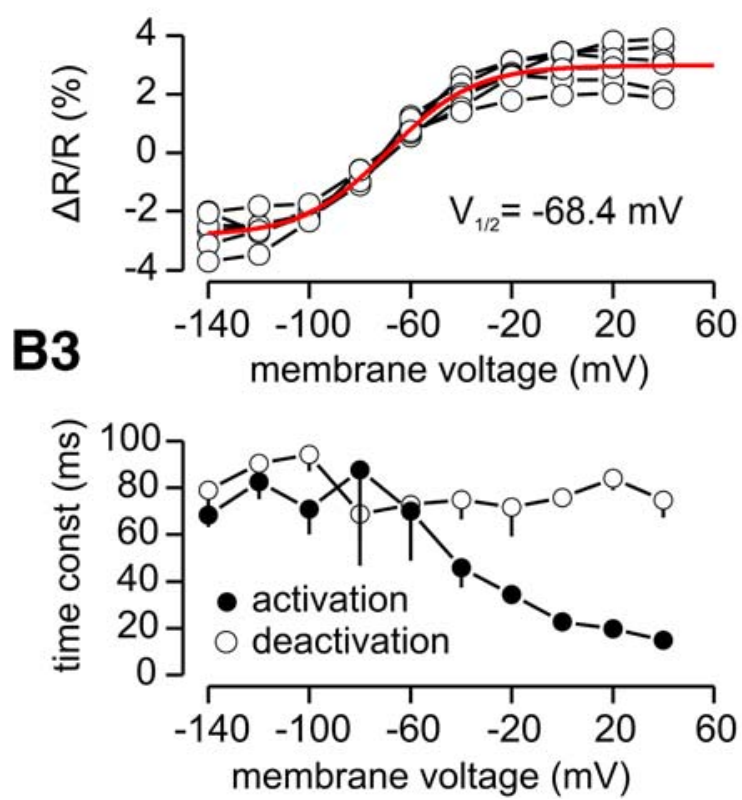

Fig. 4. Properties of VSFP2.1. Response-voltage relationship and kinetics of VSFP2.1 at $22^{\circ} \mathrm{C}(\mathrm{A} 1-\mathrm{A} 3)$ and at $35^{\circ} \mathrm{C}(\mathrm{B} 1-\mathrm{B} 3)$. (A1, B1) Ratio of yellow/cyan fluorescence during a family of $500 \mathrm{~ms}$ voltage steps from a holding potential of $-70 \mathrm{mV}$ to test potentials of -140 to $+40 \mathrm{mV}$ (20 mV increments). Traces are grand averages over average responses from 4 cells (A1) and 6 cells (B1). (A2, B2) Ratio of yellow/cyan fluorescence versus test membrane voltage. Connected symbols are data from individual cells. Red lines are Boltzmann fits with $V_{1 / 2}$ values as indicated. (A3, B3) Activation and deactivation time constants. Taken from Dimitrov et al. (2007).

shift the voltage dependency to a more physiological range, the Knöpfel laboratory investigated a series of mutational alterations in the S4 segment of Ci-VSP's voltage sensing domain. The most successful mutation was R217Q which resulted in the protein termed VSFP2.1 (Fig. 4A, B). Like the initial constructs, VSFP2.1 displayed bright fluorescence and clear targeting to the plasma membrane. VSFP2.1 exhibited a $V_{1 / 2}$ value of approximately $-70 \mathrm{mV}$ both at 22 and at $35^{\circ} \mathrm{C}$ (Fig. 4). At $22^{\circ} \mathrm{C}$, activation and deactivation kinetics were similar to those of VSFP2A-D. At $35^{\circ} \mathrm{C}$, activation was considerably faster (Fig. 4B). Figure 5 illustrates expression of VSFP2.1 (using codon optimized DNA) in a cultured cortical neuron along with neuronal responses to hyperpolarizing current injections. This experiment illustrates that, in contrast to calcium imaging, voltage imaging can directly monitor membrane hyperpolarization. The fluorescence-voltage relationship with a $V_{1 / 2}$ value in the physiological range of neuronal membrane fluctuations together with the relatively fast kinetics makes VSFP2.1 a suitable candidate for optical measurements of neuronal activity, such as large synaptic potentials; single action potentials, action potential trains; and bistabilities in resting membrane potential. To demonstrate this prediction, PC12 cells were voltage-clamped with membrane 

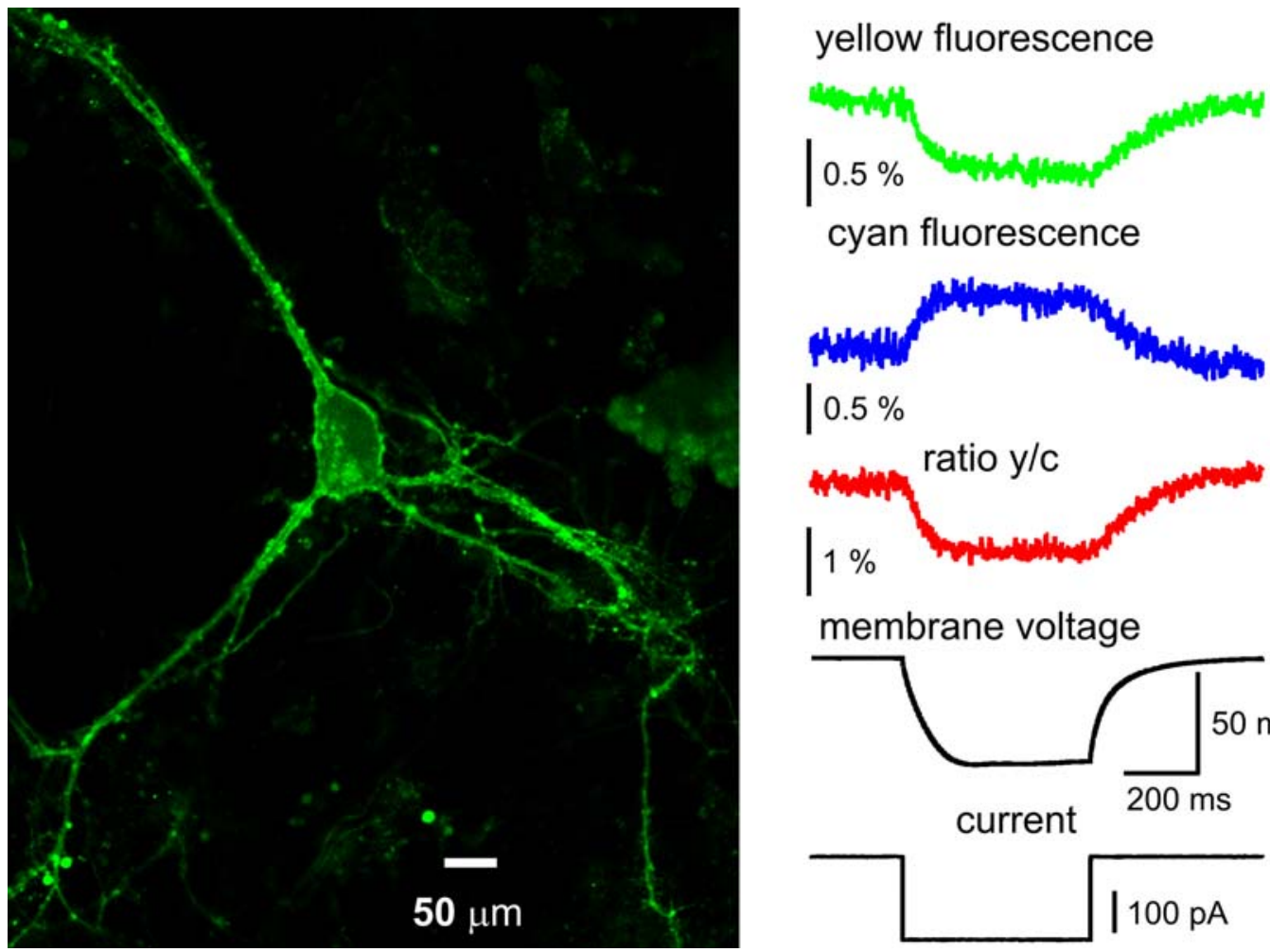

\section{cyan fluorescence}

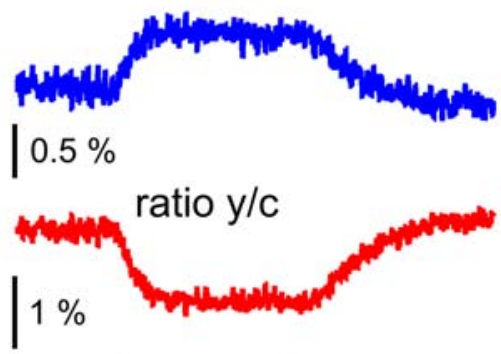

membrane voltage

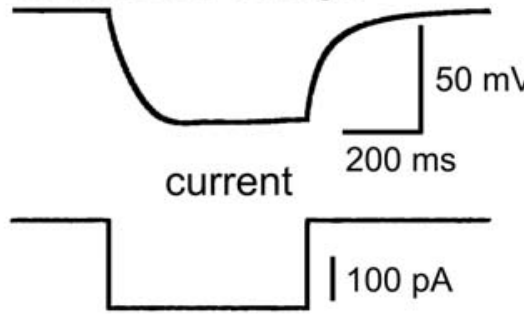

Fig. 5. Neuronal expression and functionality of VSFP2.1. Primary mouse cortical cultures were transfected with a codon optimized version of VSFP2.1 at 10 DIV. Left panel shows a pyramidal-shaped neuron expressing this FP voltage sensor. Right panel shows response of membrane potential, yellow fluorescence, and cyan fluorescence to a hyperpolarizing current injected via a patch pipette. Fluorescence values are expressed as percentage of total measured light intensity, without correction for background fluorescence (Mutoh et al., unpublished observations).

potential traces obtained from mouse mitral cells. For the trace shown in Fig. $6 \mathrm{~A}$, the mitral cell was stimulated by injection of a current pulse through a patch-clamp electrode to generate a series of action potentials. The fluorescent output of VSFP2.1 could clearly resolve individual action potentials (arrows in Fig. 6A) as well as the slower underlying membrane depolarization. As expected from the response kinetics of VSFP2.1, the optical readout of the fast action potentials was reduced relative to the slower components of the membrane potential change. This phenomenon was also clearly seen when using the response of a mitral cell to a single shock stimulation of the olfactory nerve (Fig. 6B). This response consisted of a burst of fast action potentials on a slow synaptic potential. The response of VSFP2.1 mainly represented the slow synaptic potential. More importantly, it should be noted that the responses shown in Fig. 6B could be resolved in single sweeps with a very reasonable signal-to-noise ratio (for experimental details, see Dimitrov et al., 2007).

\section{Next generation FP voltage sensors}

Further development of VSFP2.1 is progressing in several directions: (i) linker optimization, (ii) alternative FP variants, and (iii) a search for additional ways to fuse or insert FPs into Ci-VSP or alternative sensor proteins.

\section{Linker optimized variants}

It is well established for other types of FP sensors that sensitivity of the probe $(\Delta F / F$ or $\Delta R / R$ values per change in membrane voltage) can be increased by optimizing the length and amino acid composition of the linker between the sensor protein and 
A

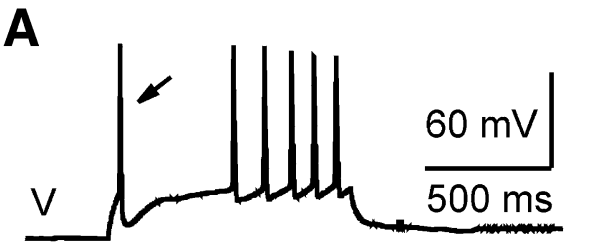

B

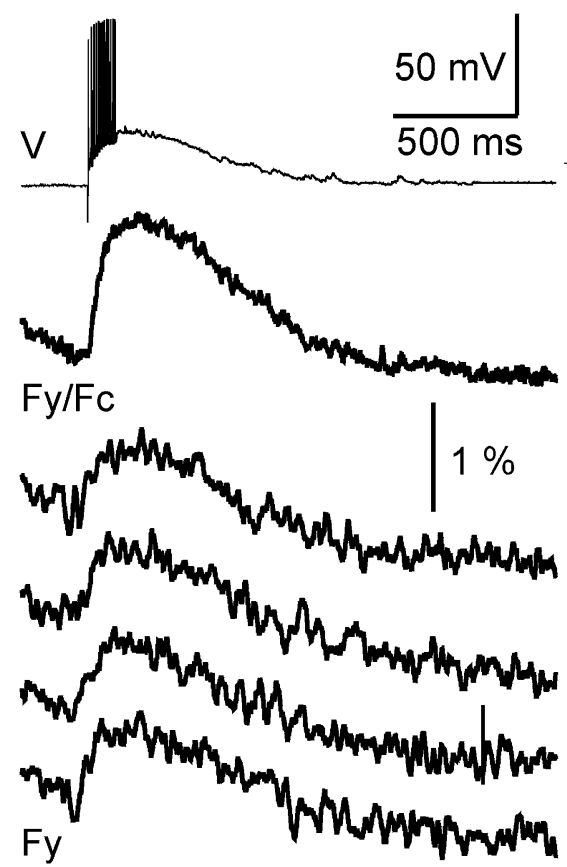

Fig. 6. VSFP2.1 can monitor physiological neuronal membrane voltage dynamics. PC12 cells expressing VSFP2.1 were voltage-clamped with a voltage trace obtained from a current-clamped mouse olfactory bulb mitral cell. The mitral cell was stimulated to generate a series of action potentials by intracellular injection of a current pulse $(A)$ or by electrical stimulation of the olfactory nerve (B). Traces in (A) are averages of 50 sweeps, upper traces (in B) are the average of 90 sweeps, and the lower four traces in (B) are single sweeps. Traces show membrane potential $(\mathrm{V})$, yellow fluorescence $(\mathrm{Fy})$, cyan fluorescence $(\mathrm{Fc})$, and the ratio of yellow and cyan fluorescence (Fy/Fc). Fluorescence signals were digitally low pass filtered $(0.2 \mathrm{kHz})$ and were not corrected for dye bleaching. Recordings were done at $35^{\circ} \mathrm{C}$. Taken from Dimitrov et al. (2007).

the FP. To such effect, the performance of VSFP2.1 was improved by removal of five amino acids that initially originated from engineered restriction sites in VSFP2.1 (Dimitrov et al., unpublished observation; Villalba-Galea et al., 2008). Further improvement of sensor sensitivity by optimizing linker is likely to be realized in near future.

\section{Alternative FP colors}

Cyan- and yellow-emitting variants of green fluorescent protein (GFP) from Aequorea victoria (CFP and YFP, respectively) are the most widely used components for FRET-based biosensors. However, there are reasons why alternative colors, particularly red shifted versions, are more desirable: (i) for deep tissue imaging, (ii) for reduced interference from tissue autofluorescence and hemoglobin absorption, and (iii) in combination with other fluorescent probes (spectral separation). Several efficient variants of these were very recently generated in the Knöpfel laboratory, the most promising comprised combinations of either a green FP with a red FP or a yellow FP with a far-red FP (Dimitrov et al., unpublished observations).

In addition, for some applications, non-FRET monochromatic probes are more favorable. Single FP sensors have been demonstrated previously including the Flash type of voltage sensors and several types of $\mathrm{Ca}^{2+}$ sensors (Baird et al., 1999; Nakai et al., 2001). Thus, we recently developed analogous single FP voltage sensors based on VSFP2s (Lundby et al., 2008; Mutoh et al., unpublished observation; Baker and Cohen, unpublished observation). The best variant characterized to date exhibited an activation time constant $(\tau$-on) of $<3 \mathrm{~ms}$ at depolarized membrane potentials (Lundby et al., 2008).

\section{Alternative designs}

In a canonical voltage sensor domain, it is not only the S4 segment that responds to voltage shifts, but rather the whole domain undergoes conformational 
changes in a complex manner (Pathak et al., 2007). It follows, therefore, that finding alternative sites for insertions of FPs along the voltage sensor domain that would not disturb the function and folding of either the voltage sensor domain or the FP may lead to new designs of improved functional voltage sensors. To explore this approach, the Hughes laboratory is using its transponson technology to generate a library of key positions in the Ci-VSP that would allow for insertion of a FP without disturbing the folding of the Ci-VSP (critical for voltage sensing), the folding of the FP itself (critical for the fluorescence report), or plasma membrane targeting.

In addition to the above there are many additional, only partially explored approaches for conformational tuning of the parts constituting the sensor. These include addition of prenylation signals or other signals for post-translational modifications that may help improve the plasma membrane targeting and/or conformational states, in a way that would be advantageous for the desired function.

\section{Genetic targeting of neurons}

Perhaps the most important feature of genetically encodable sensors is the possibility to achieve indicator loading in specific cell populations. This is the case because cell type-specific protein expression can be achieved by the use of appropriate regulatory gene sequences (promoters). This concept had been proposed many years ago in conjunction with the bioluminescent calcium-sensitive reporter protein aqueorin; however, the light output of bioluminescent probes was rather limited and consumed a co-factor that needed to be applied to the preparation. Over the last years cell type-specific expression of FP variants, or fusion proteins containing them, has been demonstrated in numerous transgenic and gene targeted mouse lines. For some experimental questions it is desirable to label cells sparsely so that individual cells can easily be identified by fluorescence imaging in densely packed tissue. Sparse labeling can be achieved with "shotgun" promoters such as Thy 1 (Feng et al., 2000), virus-based transfection (Dittgen et al., 2004), or transgenic strategies for combinatorial expression of FP components (Livet et al., 2007) but is associated with considerable uncertainty about the identity of the labeled cells and/or whether the labeled cells are representative for the given target population.

The specificity of virus-based transfection can be increased by cell-specific promoters, but this technique is limited by the size of recombinant sequence which can be packaged in to the virus. Perhaps more promising is the combination of viruses with mouse lines expressing recombinases, such as Cre and Flp, in specific subsets of neurons. This technology permits targeted expression of FP sensors in any subset neuron, for which one of the now many recombinase-mice lines are available. For that purpose the sensor gene in a lox-stop-lox cassette is inserted into a virus and then expressed under lox recombination as defined by the recombinase mouse.

A more expensive and time-consuming approach is direct transgenic or genetic targeted expression of the FP sensor. The principal advantage being reproducible preparation for experimental convenience and for a greater certainty in establishing what is stained (and-equally important-what is not). With such genetic models the precise targeting allows optical signals to be reliably attributed to specific subsets of cells within a network consisting of many different cell types, whilst also allowing low noise imaging to be performed without optically resolving the labeled cells (Knöpfel et al., 2006; Díez-García et al., 2005, 2007; Qiu and Knöpfel, 2007).

\section{Genetically encoded sensors of membrane potential compared to alternative genetic targeting approaches for fluorescence voltage-sensitive probes}

In addition to voltage sensors that are entirely genetically encoded, at least two other approaches to overcoming indiscriminant dye staining use genetics in combination with conventional organic chromophores. The first is a hybrid approach that combines the advantages of conventional voltagesensitive dyes (relatively large $\Delta F / F$ ) with genetic targeting (Chanda et al., 2005; DiFranco et al., 2007). The hybrid probe hVOS employs FRET between membrane-anchored GFP and a dye, 
DPA, that partitions into the plasma membrane in a membrane voltage-dependent manner. While this hybrid probe produces the largest reported $\Delta F / F$ values among all genetically encoded voltagesensitive probes, the high concentration of the lipophilic anion DPA has a significant effect on the activity of excitable membranes (Fernández et al., 1983; Chanda et al., 2005; Zimmermann et al., 2008). Thus reduced DPA concentrations were required to obtain action potential responses (Chanda et al., 2005) resulting in much smaller fractional changes $(\Delta F / F)$.

Using an optimized DPA-based voltage reporter, Sjulson and Miesenböck (2008) found a failure to detect action potentials in the Drosophila antennal lobe, presumably because spike initiation and/or propagation are inhibited by the capacitive load added even at reduced DPA membrane densities.

The second semi-genetic method uses the genetically targetable expression of an enzyme with an organic dye as a substrate. The idea is that only the enzymatically processed dye stains the plasma membrane where it functions as a conventional voltage-sensitive dye. In a proof of principle study (Hinner et al., 2006), a lipophilic dye was made water-soluble by the addition of phosphate groups to the hydrophobic tail. Cellspecific staining was achieved by genetically expressing an outward-facing membrane-bound phosphatase that cleaves the hydrophilic phosphate group from the engineered dye, resulting in a closely localized population of lipophilic product dye that integrates and stains the membrane of the specified target cells. While this approach has potential and essentially could be used with a variety of established voltage-sensitive lipid dyes, in its current state the applicability in neuronal tissues is unproven. Several obstacles become apparent; first, the usual issues of dye accessibility to the area of study and dye toxicity. Second, a question arises whether the phosphatase would not catalyze intrinsic processes disturbing the physiological state of the tissue, or the dye itself would not become substrate to intrinsic proteins resulting in unwanted staining. Third, in a tissue where the individual cells are closely packed together, the "released" dye is expected to diffuse and bind to plasma membranes of "not-selectedfor" cells that are adjacent to the cell expressing the enzyme.

\section{Signal-to-noise considerations}

The motivation for the development of FP voltage sensors is to overcome unselective labeling obtained when staining tissue with organic voltagesensitive dyes. Selective staining of specific subsets of neurons will allow a more stringent interpretation of optically monitored voltage signals, in terms of their source. The second, similarly important benefit is an increase in $\mathrm{S} / \mathrm{N}$ ratio because undesired staining contributes to noise but not to signal. In this context, it was as a consequence of unspecific labeling (in the form of limited membrane targeting) that the first generation of FP voltage sensors failed. The second generation overcame this problem. Yet, the signal-to-noise ratio $\left(R_{\mathrm{S} / \mathrm{N}}\right)$ is probably the most critical parameter, particularly when a high temporal resolution is required. For in vivo experiments a low $R_{\mathrm{S} / \mathrm{N}}$ often results from a contamination of the sensor signal by movementinduced changes in the measured light intensity, for instance: changes in blood volume or hemoglobin oxygenation, light scattering or endogenous tissue fluorescence (for a recent review, see Baker et al., 2005). Synchronization with breathing and/or heartbeat along with careful selection of excitation and emission wavelengths can reduce these noise signals significantly (Baker et al., 2005).

There is, however, a physical limit for $R_{\mathrm{S} / \mathrm{N}}$ in optical recordings due to the statistics of photon detection (expanded in Knöpfel et al. 2006 and Sjulson and Miesenböck, 2007). Thus, in the best case, $R_{\mathrm{S} / \mathrm{N}}$ is increased with the square root of the number of photons detected per temporal and spatial bin. Accordingly, it can be predicted that the first successful (because least demanding) application of FP voltage sensors in intact tissue will be voltage imaging from populations of subsets of neurons, as previously demonstrated with FP calcium sensors (Díez-García et al., 2005, 2007). Spatial averaging over many neurons that carry a common voltage signal will provide a sufficient emission intensity to detect small modulations (Vranesic et al., 1994). $R_{\mathrm{S} / \mathrm{N}}$ decreases with the sampling rate (as the number of sampled photon decreases with shorter sampling intervals, but also because of an increased contribution of noise resulting from the sampling process). For these reasons, along with the fact that imaging sampling frequency is limited for some imaging techniques (like laser scanning), an FP voltage 
sensor with somewhat slow response characteristics may be more suitable for certain applications. Indeed, slow response kinetics act as a signal conditioning mechanisms (as required by the sampling theorem). Yet, timing information may be recovered from slowly responding indicators by deconvolution techniques (Yaksi and Friedrich, 2006).

\section{Capacitive load and other possible caveats}

Genetically encoded optical sensors make it possible to record from multiple cells in undamaged tissue. However, as a recombinant gene product, the sensor itself can interfere with intrinsic biochemical and/or physical cell functions. Hence, as in the case of any artificially introduced probe, FP sensor-derived data require verification against controls to demonstrate the absence of probeinduced undesired effects. Unlike synthetic potentiometric probes, e.g. cyanin and oxonal dyes, which are notorious for their phototoxicity (Grinvald et al., 1982; Kalyanaraman et al., 1987), FP-based sensors are not known, and are not expected, to form toxic illumination-induced products. Similar to synthetic probes, however, they add to the nonlinear dielectric polarization of the membrane resulting in increased membrane "capacitive load" (Sjulson and Miesenböck, 2007). The effect is based on a simple physical mechanism: The voltage sensor domain moves positive charges across the membrane in response to changes in membrane potential. This charge movement manifesting as a transmembrane capacitive current (well known as gating currents for ion channels) redistributes charge between the inner and outer membrane surface in a voltage sensor domain state-dependent manner, tantamount to a voltageand time-dependent nonlinear capacitance. Expression of voltage sensor domain-containing proteins like VSFPs therefore contributes an extra capacitance to the membrane. Depending on its magnitude and activation time course, this extra capacitance may influence the subthreshold and spiking properties of target membranes. The physiological effect of extra mobile membrane charge can be inferred from the behavior of excitable membranes after uptake of lipophilic anions, like dipicrylamine (DPA), causing a dose-dependent amplification of membrane capacitance and, at higher doses, decrement of action potential amplitude, increased action potential width, slowing of spike propagation (Fernández et al., 1983), and reduced field potentials (Chanda et al., 2005). As the biophysical mechanism is fairly basic, simulation models can be used to predict and delimit the effects of extra mobile charges on the electrical response properties of excitable membranes (Fernández et al., 1983; DiFranco et al., 2007).

\section{Future directions}

In this review we have focused on the most advanced approach for FP voltage sensor engineering based on a fusion between a voltage sensor protein and an FP reporter domain. However, an alternative design of an FP voltage sensor might be to use the FP as both the sensor and reporter. Such a protein would have the smallest possible size and minimal interaction with other organism-intrinsic proteins. To achieve this, the FP would need to be localized at the water-lipid interface of the plasma membrane or even largely within the lipid phase. Sensitivity to membrane voltage could be based on either voltage-dependent changes in conformation and lipid-protein interactions or be a direct effect of the transmembrane electric field on the optical properties of the chromophore (Molecular Stark effect). The challenge, however, is to change the amino acid composition of the FP molecule so that it would intrinsically translocate to the hydrophobic phase without interruption to the maturation, constitution, and existence of a functional chromophore.

In conclusion, FP sensors will open paths to yet unexplored territories of functional neuroimaging. In addition to the FP voltage sensors on which this review focuses, there are now many different FP sensors for monitoring biochemical processes and transmitter dynamics (Tsien 2005), which can be applied to neuronal circuits. Reading neuronal circuit activity can also be complemented with emerging optical methods to write activity into neuronal circuits (Miesenbock and Kevrekidis, 2005; Boyden et al., 2005; Knöpfel 2008). In the future these techniques may be combined to yield a bidirectional optical interface to brain function. 


\section{Acknowledgments}

The authors thank Dr. Steven Middleton for careful commenting on this article. This article was supported by NIH grant NS057631 (LBC, EYI, VAP, $\mathrm{TH}, \mathrm{TK}$ ) and an intramural grant from RIKEN BSI (TK).

\section{Open Access}

This article is distributed under the terms of the Creative Commons Attribution Noncommercial License which permits any noncommercial use, distribution, and reproduction in any medium, provided the original author(s) and source are credited.

\section{References}

Ataka, K. and Pieribone, V. A. (2002). A genetically targetable fluorescent probe of channel gating with rapid kinetics. Biophys. J. 82, 509-516.

Baird, G. S., Zacharias, D. A., and Tsien, R. Y. (1999). Circular permutation and receptor insertion within green fluorescent proteins. Proc. Natl. Acad. Sci. USA 96, 11241-11246.

Baker, B. J., Kosmidis, E. K., Vucinic, D., Falk, C., Cohen, L., Djurisic, M., and Zecevic, D. (2005). Imaging brain activity with voltage- and calcium-sensitive dyes. Cell. Mol. Neurobiol. 25, 245-282.

Baker, B. J., Lee, H., Pieribone, V. A., Cohen, L. B., Isacoff, E. Y., Knöpfel, T., and Kosmidis, E. K. (2007). Three fluorescent protein voltage sensors exhibit low plasma membrane expression in mammalian cells. J. Neurosci. Methods 161, 32-38.

Brown, J. E., Cohen, L. B., De Weer, P., Pinto, L. H., Ross, W. N., and Salzberg, B. M. (1975). Rapid changes in intracellular free calcium concentration. Detection by metallochromic indicator dyes in squid giant axon. Biophys. J. 15, 1155-1160.

Boyden, E. S., Feng, J., Bamberg, E., Nagel, G., and Deisseroth, K. (2005). Millisecond-timescale, genetically targeted optical control of neural activity. Nat. Neurosci. 8, 1263-1268.
Chanda, B., Blunck, R., Faria, L. C., Schweizer, F. E., Mody, I., and Bezanilla, F. (2005). A hybrid approach to measuring electrical activity in genetically specified neurons. Nat. Neurosci. 8 , 1619-1626.

Davila, H. V., Salzberg, B. M., Cohen, L. B., and Waggoner, A. S. (1973). A large change in axon fluorescence that provides a promising method for measuring membrane potential. Nat. New Biol. 241, 159-160.

Díez-García, J., Akemann, W., and Knöpfel, T. (2007). In vivo calcium imaging from genetically specified target cells in mouse cerebellum. Neuroimage 34, 859-869.

Díez-García, J., Matsushita, S., Mutoh, H., Nakai, J., Ohkura, M., Yokoyama, J., Dimitrov, D., and Knöpfel, T. (2005). Activation of cerebellar parallel fibers monitored in transgenic mice expressing a fluorescent $\mathrm{Ca}^{2+}$ indicator protein. Eur. J. Neurosci. 22, 627-635.

DiFranco, M., Capote, J., Quiñonez, M., and Vergara, J. L. (2007). Voltage-dependent dynamic FRET signals from the transverse tubules in mammalian skeletal muscle fibers. J. Gen. Physiol. 130, 581-600.

Dimitrov, D., He, Y., Mutoh, H., Baker, B. J., Cohen, L., Akemann, W., and Knöpfel, T. (2007). Engineering and characterization of an enhanced fluorescent protein voltage sensor. PLoS ONE 2(5), e440. doi:10.1371/journal.pone. 0000440 .

Dittgen, T., Nimmerjahn, A., Komai, S., Licznerski, P., Waters, J., Margrie, T. W., Helmchen, F., Denk, W., Brecht, M., and Osten, P. (2004). Lentivirus-based genetic manipulations of cortical neurons and their optical and electrophysiological monitoring in vivo. Proc. Natl. Acad. Sci. USA 101, 18206-18211.

Feng, G., Mellor, R. H., Bernstein, M., Keller-Peck, C., Nguyen, Q. T., Wallace, M., Nerbonne, M. N., Lichtman, J. W., and Sanes, J. R. (2000). Imaging neuronal subsets in transgenic mice expressing multiple spectral variants of GFP. Neuron 28, 41-51.

Fernández, J. M., Taylor, R. E., and Bezanilla, F. (1983). Induced capacitance in the squid giant axon. Lipophilic ion displacement currents. J. Gen. Physiol. 82, 331-346.

Grinvald, A. and Hildesheim, R. (2004). VSDI: a new era in functional imaging of cortical dynamics. Nat. Rev. Neurosci. 5, 874-885. 
Grinvald, A., Hildesheim, R., Farber, I. C., and Anglister, L. (1982). Improved fluorescent probes for the measurement of rapid changes in membrane potential. Biophys. J. 39, 301-308.

Guerrero, G., Siegel, M. S., Roska, B., Loots, E., and Isacoff, E. Y. (2002). Tuning FlaSh: redesign of the dynamics, voltage range and color of the genetically-encoded optical sensor of membrane potential. Biophys. J. 83, 3607-3618.

Hinner, M. J., Hübener, G., and Fromherz, P. (2006). Genetic targeting of individual cells with a voltage-sensitive dye through enzymatic activation of membrane binding. ChemBioChem 7, 495-505.

Jiang, Y., Lee, A., Chen, J., Ruta, V., Cadene, M., Chait, B. T., and MacKinnon, R. (2003). X-ray structure of a voltage-dependent $\mathrm{K}^{+}$channel. Nature 423, 33-41.

Jiang, Y., Ruta, V., Chen, J., Lee, A., and MacKinnon, R. (2003). The principle of gating charge movement in a voltage-dependent $\mathrm{K}+$ channel. Nature 423, 42-48.

Kalyanaraman, B., Feix, J. B., Sieber, F., Thomas, J. P., and Girotti, A. W. (1987). Photodynamic action of merocyanine 540 on artificial and natural cell membranes: involvement of singlet molecular oxygen. Proc. Natl. Acad. Sci. USA 84, 2999-3003.

Knöpfel, T. (2008). Expanding the toolbox for remote control of neuronal circuits. Nat. Methods 5, 293-295.

Knöpfel, T., Díez-García, J., and Akemann, W. (2006). Optical probing of neuronal circuit dynamics: genetically encoded versus classical fluorescent sensors. Trends Neurosci. 29, 160-166.

Knöpfel, T., Tomita, K., Shimazaki, R., and Sakai, R. (2003). Optical recordings of membrane potential using genetically targeted voltage-sensitive fluorescent proteins. Methods 30, 42-48.

Kohout, S. C., Ulbrich, M. H., Bell, S. C., and Isacoff, E. Y. (2007). Subunit organization and functional transitions in Ci-VSP. Nat. Struct. Mol. Biol. 15, 106-108.

Livet, J., Weissman, T. A., Kang, H., Draft, R. W., Lu, J., Bennis, R. A., Sanes, J. R., and Lichtman, J. W. (2007). Transgenic strategies for combinatorial expression of fluorescent proteins in the nervous system. Nature 450, 56-62.

Long, S. B., Campbell, E. B., and MacKinnon, R. (2005). Crystal structure of a mammalian voltage-dependent Shaker family $\mathrm{K}^{+}$channel. Science 309, 897-903.

Long, S. B., Campbell, E. B., and MacKinnon, R. (2005). Voltage sensor of Kv1.2: structural basis of electromechanical coupling. Science 309, 903-908.

Lundby, A., Mutoh, H., Dimitrov, D., Akemann, W., and Knöpfel, T. (2008). Engineering of a genetically encodable fluorescent voltage sensor exploiting fast Ci-VSP voltage-sensing movements. PLoS ONE 3, e2514.

MacDonald, V. W. and Jobsis, F. F. (1976). Spectrophotometric studies on the $\mathrm{pH}$ of frog skeletal muscle. $\mathrm{pH}$ change during and after contractile activity. J. Gen. Physiol. 68, 179-195.

Miesenbock, G. and Kevrekidis, I. G. (2005). Optical imaging and control of genetically designated neurons in functioning circuits. Annu. Rev. Neurosci. 28, 533-563.

Murata, Y., Iwasaki, H., Sasaki, M., Inaba, K., and Okamura, Y. (2005). Phosphoinositide phosphatase activity coupled to an intrinsic voltage sensor. Nature 435, 1239-1243.

Nakai, J., Ohkura, M., and Imoto, K. (2001). A high signal-to-noise $\mathrm{Ca}^{2+}$ probe composed of a single green fluorescent protein. Nat. Biotechnol. 19, 137-141.

Pathak, M. M., Yarov-Yarovoy, V., Agarwal, G., Roux, B., Barth, P., Kohout, S., Tombola, F., and Isacoff, E. Y. (2007). Closing in on the resting state of the Shaker $\mathrm{K}+$ channel. Neuron 56, 124-140.

Perozo, E., MacKinnon, R., Bezanilla, F., and Stefani, E. (1993). Gating currents from a nonconducting mutant reveal open-closed conformations in Shaker K+ channels. Neuron 11, 353-358.

Qiu, D. L. and Knöpfel, T. (2007). An NMDA receptor/nitric oxide cascade in presynaptic parallel fiber-Purkinje neuron long-term potentiation. J. Neurosci. 27, 3408-3415.

Ramsey, S. I., Moran, M. M., Chong, J. A., and Clapman, D. E. (2006). A voltage-gated protonselective channel lacking the pore domain. Nature 440, 1213-1216.

Sakai, R., Repunte-Canonigo, V., Raj, C. D., and Knöpfel, T. (2001). Design and characterization of a DNA-encoded, voltage-sensitive fluorescent protein. Eur. J. Neurosci. 13, 2314 2318.

Sasaki, M., Takagi, M., and Okamura, Y. (2006). A voltage sensor-domain protein is a voltage- 
gated proton channel. Science 312(5773), 589-592.

Siegel, M. S. and Isacoff, E. Y. (1997). A genetically encoded optical probe of membrane voltage. Neuron 19, 735-741.

Sjulson, L. and Miesenböck, G. (2007). Optical recording of action potentials and other discrete physiological events: a perspective from signal detection theory. Physiology 22, 47-55.

Sjulson, L. and Miesenböck, G. (2008). Rational optimization and imaging in vivo of a genetically encoded optical voltage reporter. J. Neurosci. 28, 5582-5593.

Tombola, F., Ulbrich, M., and Isacoff, E. Y. (2008). The voltage-gated proton channel Hv1 has two pores each controlled by one voltage sensor. Neuron 58(4), 546-556.

Tsien, R. Y. (2005). Building and breeding molecules to spy on cells and tumors. FEBS Lett. 579, 927-932.

Villalba-Galea, C. A., Dimitrov, D., Mutoh, H., Lundby, A., Sadntner, W., Bezanilla, F., and
Knöpfel, T. (2008). Charge movement of the voltage sensitive fluorescent protein. Biophys. J. 94, 1362.

Vranesic, I., lijima, T., Ichikawa, M., Matsumoto, G., and Knöpfel, T. (1994). Signal transmission in the parallel fiber-Purkinje cell system visualized by high-resolution imaging. Proc. Natl. Acad. Sci. USA 91, 13014-13017.

Yaksi, E. and Friedrich, R. W. (2006). Reconstruction of firing rate changes across neuronal populations by temporally deconvolved $\mathrm{Ca}^{2+}$ imaging. Nat. Methods 3(5), 377-383.

Zimmermann, D., Kiesel, M., Terpitz, U., Zhou, A., Reuss, R., Kraus, J., Schenk, W. A., Bamberg, E., and Sukhorukov, V. L. (2008). A combined patch-clamp and electrorotation study of the voltage- and frequency-dependent membrane capacitance caused by structurally dissimilar lipophilic anions. J. Membr. Biol. 221, 107-121. 